\title{
Condicionamientos multilaterales a la reforma venezolana en salud
}

\section{Gamboa Cáceres, Teresa*}

\section{Resumen}

Se analiza la influencia de los organismos multilaterales en la reforma del sistema de salud en Venezuela, cuyo modelo formal acaba de formularse legalmente, aun cuando la ejecución se viene adelantando hace algunos años. Entre los factores que orientan el sentido del cambio se considera el papel de entidades como el Banco Interamericano de Desarrollo y el Banco Mundial, los cuales condicionan los préstamos a la puesta en práctica de ciertas reformas por parte del país receptor; el principal centro de atención son los cambios vinculados más directamente con la adecuación del sistema de salud a una economía de mercado, de acuerdo con patrones avalados internacionalmente, cuyos rasgos principales se precisan en este informe, para ser contrastados con las exigencias de los organismos multilaterales de acuerdo a los convenios suscritos. Como estrategias fundamentales se encontraron la reducción del gasto social en salud, la descentralización como vía para la consolidación de la autonomía legal y operativa de los centros de atención (hospitales y ambulatorios) y su posterior privatización, así como la reconversión laboral con tendencia a la flexibilización del contrato de trabajo. Estos factores deberian presionar hacia la eficiencia, pero no aseguran la universalidad ni la equidad en la prestación de los servicios de salud.

Palabras clave: Banco Mundial, Banco Interamericano de Desarrollo, Reforma Administrativa del Estado, Sistemas de Salud, Venezuela.

\section{Recibido: 98-11-23 . Aceptado: 99-02-17}

- Investigadora del Centro de Estudios de la Empresa (Facultad de Ciencias Económicas y Sociales) de la Universidad del Zulia, Maracaibo. Magister en Evaluación de la Gestión de Empresas Públicas y aspirante al Doctorado en Ciencias Politicas. Es investigadora acreditada en el Programa de Promoción del Investigador (PPI) del Consejo Nacional de Investigaciones Científicas y Tecnológicas. 


\section{Multilateral Conditionings in Venezuelan Health Reform}

\section{Abstract}

The influence of multilateral organisms in Venezuelan health reform is analyzed, the model for which has just been legally finished, even though its execution began several years ago. Among the factors which orient this sense of change, we consider the role of entities such as the Interamerican Development Bank and the World Bank, which condition their loans on the execution of certain reform practices on the part of the receiving country; the principal centers of attention are the changes directly related to the adjustment of the health system to a market economy according to internationally approved patterns, the characteristics of which are explained in this paper, and which can be contrasted with the requirements of multilateral organisms according to agreed upon covenants. As fundamental strategies we find the reduction of social health costs, decentralization in order to consolidate legal and operative autonomy in health centers (hospitals and ambulatory units) and their eventual privatization, reconversion of the work force and more flexibility in labor contracts. These factor should create pressure for more efficiency, but do not guarantee universality nor equality in the health services offered.

Key words: World Bank, Interamerican Development Bank, administrative state reform, health systems, Venezuela.

\section{Introducción}

En el contexto de los procesos de modernización de la gestión pública, desencadenados por la crisis fiscal y por la globalización e informatización de la sociedad, nos proponemos en este trabajo explorar la incidencia de los organismos multilaterales en la definición de los rasgos fundamentales de la reforma del sistema de salud en Venezuela.

Partimos de considerar que los cambios en la gestión pública de la salud que se están produciendo en América Latina, aún guardando la especificidad indispensable para adecuarse a cada contexto, presentan algunas características comu- nes, que coinciden además con los aspectos fundamentales de la modernización que los países occidentales de Europa y Estados Unidos han desarrollado.

En tal sentido, manejamos la siguiente hipótesis: el factor unificador de la reforma económica y administrativa en países de distintas regiones, está directamente vinculado a la intervención de los organismos multilaterales, especialmente los de carácter financiero, aún cuando también cuentan con el apoyo técnico de los organismos científicos y/o de formación. Los primeros, como requisito para el otorgamiento de préstamos o apoyo financiero con cargo a fondos perdidos, 
establecen los objetivos del cambio administrativo, especialmente en lo relativo a la orientación hacia la economia de mercado y la política de personal con miras a la racionalización de costos; asi mismo prestan la asesoría necesaria.

Entre las entidades de apoyo técnico vale destacar el Programa de las $\mathrm{Na}$ ciones Unidas para el Desarrollo (PNUD), constituido en 1966 como una de las principales fuentes de financiación de la ONU, aunque actualmente las gobernaciones pagan por su asesoria técnica (PNUD/FONVIS, 1996). Así mismo, es relevante el papel de entidades como el Centro Latinoamericano de Administración para el desarrollo (CLAD, proyecto conjunto de los gobiemos latinoamericanos y España, con sede en Venezuela), el Instituto Centroamericano de Administración Pública (ICAP), el Centro Africano de Administración Pública (CAFRAP) y la Organización Panamericana de la Salud (OPS).

Los institutos de formación han sido vehículos relevantes para la diseminación de las nuevas técnicas y herramientas gerenciales que era necesario introducir pero no lograron mejorar los sistemas administrativos (Pérez Salgado,1997,264). Su papel se evidencia por ejemplo en la naturaleza de los concursos de ensayos; e| XX| Concurso organizado conjuntamente por CLAD-OPS (1998), tenía como tema "Hacia la implantación de modelos de administración gerencial en salud pública". Los ensayos, desde una perspectiva teórica o práctica debían referirse a la transformación de la cultura, los estilos y la práctica de gestión de la salud, reflejando los procesos de modernización y privilegiando el enfoque gerencial, cuyo modelo expresa justamente los cambios que se están promoviendo internacionalmente. Además el CLAD es un valioso centro de información en la materia y en sus Congresos sobre Reforma del Estado y de la Administración Pública, facilita el encuentro de académicos y funcionarios públicos. Ambas instituciones participan directamente en la planificación y ejecución de actividades de formación en el campo administrativo.

Ahora bien, los organismos financieros multilaterales insisten en políticas de ajuste económico, en focalización de la ayuda a los sectores más desfavorecidos y en cambios en la gestión estatal que parecen producir mayor desempleo $y$ deterioro de las condiciones de empleo, a pesar de que, como afirma Kliksberg (1994a,8):

"La explosión de la pobreza está ligada a factores estructurales. Entre ellos diversas fuentes coinciden en destacar: la distribución muy desigual de los efectos de la crisis económica que a través de políticas de ajuste ortodoxas han recaído especiaimente en los sectores más desfavorecidos, las estructuras extremadamente asimétricas de distribución del ingreso, las debilidades de la política social, los ataques permanentes de ciertos sectores influyentes sobre la misma legitimidad del gasto social ... El Programa de Naciones Unidas para el Desarrollo destaca que ... la ausencia de compromiso político y no la falta de recursos financieros es con frecuencia la verdadera causa del abandono en que se encuentra el hombre".

En consecuencia, el análisis de la Reforma del Estado promovida por los organismos multilaterales, debe permitir- 
nos destacar hasta qué punto las modificaciones acordadas contienen elementos potenciales de desarrollo social, tanto desde el punto de vista del personal que labora en la administración pública, como respecto a los usuarios de los servicios, especificamente los relativos al sector salud.

Como una primera aproximación, este trabajo pretende en primer lugar, precisar los rasgos comunes de la reforma de la gestión pública en diferentes países y su concreción en el caso de los sistemas de salud en Venezuela; en segundo lugar aspira caracterizar la intervención de los organismos financieros multilaterales en el proceso de modernización administrativa en el campo de la salud pública, los objetivos que persiguen y la influencia que logran en la definición del tipo de modificaciones que se están realizando en el sistema de salud venezolano, destacando las implicaciones de dichos cambios.

\section{El Banco Mundial:}

\section{sus políticas y su relación con otros entes multilaterales}

El Banco Internacional de Reconstrucción y Fomento (BIRF) es la primera y la más importante de las instituciones que conforman la red habitualmente conocida como Banco Mundial; un país necesita ser miembro del BIRF para integrar cualquiera de las otras organizaciones de la red. EI BIRF junto con el Fondo Monetario Internacional (FMI) nacen de los convenios de Bretton Woods, inmediatamente después de la segunda guerra mundial, hecho que puso de manifiesto la hegemonia estadounidense frente a la inglesa, pero legitimada en instituciones multilaterales, que se proyectan como organismos de cooperación internacional. Sus políticas definen "un código de conducta" para las políticas económicas de los palses con déficits en la balanza de pagos, precisando ajustes concretos que tales países debe realizar.

Dado que el BIRF sólo puede financiar proyectos garantizados por gobiernos, posteriormente se crearon otros organismos, que permitieran operar en forma más amplia y diversificada: En 1956 nace la Corporación Financiera Internacional (CFI), para incentivar la inversión directamente productiva. La CFI, además de préstamos, realiza inversiones en acciones que luego traspasa al sector privado. A fines de los años cincuenta, la presión por acceder a préstamos blandos, junto con la ocurrencia de cambios sociales radicales, propiciaron la creación de la Asociación Internacional de Fomento (AIF), la cual obtiene sus fondos de ganancias del BIRFy de donaciones de países desarrollados. A mediados de los 60 , a raiz de expropiaciones y otros conflictos entre gobiernos y empresas extranjeras, se constituyó el Centro Internacional de Arreglo de Diferencias Relativas e Inversiones (C|ADI), con el objetivo de establecer ciertas reglas de conducta entre inversionistas extranjeros y estados, más que mediar en conflictos ya declarados. Finalmente, en 1985 se aprobó la creación de la Multilateral investment Guarantee Agency (MIGA), como aseguradora multilateral de las inversiones extranjeras. (Lichtensztejn y Baer, 1987,2431,136).

Aun cuando el BM tiene una dirección colegiada, en la cual la Junta de Go- 
bernadores ocupa la escala superior, seguida de los directores ejecutivos (con un poder de voto proporcional al capital y con predominio en la participación de los países desarrollados), la mayor influencia en las decisiones reside en la presidencia y sus cuadros operativos. La presidencia del $B M$ es ocupada habitualmente por un norteamericano, con frecuencia vinculado a la banca $o$ al sector productivo (mientras un europeo es director del FMI); igualmente, el personal técnico y los consultores es predominantemente de origen estadounidense, cuyo país recibe más del $50 \%$ de los trabajos de consultoría. Sin embargo, la relativa autonomía del BM y la presencia de otras fuerzas en la estructura de poder no permiten suponer una identificación absoluta entre el banco y los intereses de los E.U., cuyo comportamiento en las últimas décadas -signadas por las restricciones financieras- tiene como fin aumentar su influencia al más bajo costo posible, especialmente en lo relativo a la promoción de la economia de mercado, garantizando la apertura comercial, productiva y financiera, asignando preferencia a los países estratégicos para la dinámica capitalista (Lichtensztejn y Baer, 1987).

Los temas en los cuales ha habido oposición de E.U. al BM se refieren básicamente a: solicitudes de mayores fondos, destino y condiciones de los préstamos otorgados por el banco, especialmente cuando se trata de países con las siguientes caracteristicas: a) proclives al socialismo; b) cuya política enfrenta de alguna forma los intereses de E.U.; c) naciones con demasiada injerencia estatal y d) financiamiento para la producción energética, que en opinión de Estados
Unidos debe ser promovida por la inversión privada.

La hipótesis de Lichtensztejn y Baer sobre el desarrollo del Banco Mundial (BM) y del Fondo Monetario Internacional (FMI) como "un proceso que acompaña y contribuye a la moderna internacionalización del sistema económico, o sea que se inscribe en la nueva fase del capitalismo transnacional que se dinamiza en la segunda postguerra", sigue teniendo validez cuando nos aproximamos al año 2000. "La crisis ha servido para dejar al desnudo y sin falsos pudores las fuerzas en juego y las funciones reales que gobiernan a estas dos grandes instituciones internacionales del capitalismo transnacional"

Hasta el momento de su estudio (1987), Lichtensztejn y Baer distinguen tres etapas bien definidas en la evolución de las políticas del Banco Mundial:

Inicialmente la función fundamental del BM era la reconstrucción, más que el desarrollo, aunque su participación en el financiamiento del Plan Marshall para la reconstrucción de los principales paises capitalistas europeos después de la segunda guerra mundial fue poco significativa (inferior al 2\%). Hasta mediados de la década de los sesenta, el BM dirigía todos sus préstamos a los países desarrollados y sólo cumplió una función de apoyo a la actuación de las empresas y bancos transnacionales, mientras el FMI representaba la institución más relevante de la estructura financiera internacional.

A fines de los sesenta y durante la siguiente década, frente a la crisis monetaria, los problemas energéticos y la pérdida de legitimidad del FMI (que lo obligaron a relativizar sus esquemas cortopla- 
cistas), el BM asumió un rol más importante en la búsqueda de mecanismos para administrar la crisis, poniendo en juego una mayor audacia teórica e ideológica. En esta época se produce una cierta superposición de funciones entre el Fondo y el Banco. A partir de 1968 el BM concentró casi todos sus recursos en los paises subdesarrollados, especialmente para financiar proyectos de infraestructura con el objetivo declarado de favorecer su crecimiento económico. Este vuelco es producido por: a) la expansión y consolidación de las economías europeas y japonesas; $b$ ) la fuerte presencia de países subdesarrollados en el FMI y en el $\mathrm{BM}$, a raiz del triunfo de las luchas de liberación nacional y descolonización; y c) finalmente, por la reducción de la capacidad de ayuda externa de E.U. A partir del informe Pearson en 1969, el Banco se plantea nuevas tareas, para enfrentar los desequilibrios sociales internos de los países subdesarrollados - vinculadas a la satisfacción de las necesidades básicas (pobreza)- lo cual impulsó el estudio de las realidades específicas de estos países y el desarrollo de técnicas de evaluación de políticas económicas y sociales. Sin embargo, el BM favoreció primordialmente la marcha de la economía internacional en lo relativo a desarrollo energético y ajustes estructurales en las inversiones públicas y en los sectores manufactureros y de exportación, en tanto atañen a la articulación de las fuerzas productivas de tales naciones con la actividad industrial de los países centrales.

Desde 1982, con el desencadenamiento de la crisis de endeudamiento y gracias a la creciente interacción entre el FMl y el BM, se incrementan las acciones combinadas y complementarias, centradas en los Países del Tercer Mundo, desarrollando programas de ajuste estructural en función de construir soluciones al pago de la deuda externa y cofinanciamiento de proyectos contra el subdesarrollo, pero en el marco de la expansión de los capitales internacionales. Se trata de apoyar financieramente los costos de transición relativos a cambios concretos en las políticas públicas para facilitar reformas estructurales, pero también actuar como catalizador de la inversión externa.

A las etapas anteriores deberíamos agregar las siguientes, no abarcadas por el estudio de Lichtensztejn y Baer:

El viraje hacia la atención prioritaria de los países antes socialistas, a raíz de los cambios geopoííticos producidos a finales de los ochenta por el colapso y desintegración de la Unión Soviética. Se trata de favorecer la integración a la economía capitalista de estos nuevos mercados, asi como tomar las previsiones para evitar un "retroceso" hacia el modelo socialista.

A finales de la década del noventa tanto el Banco Interamericano de Desarrollo (BID) como el BM en sus respectivos informes de 1997 reconocen que los intentos de desarrollo basados en el protagonismo del Estado y las reformas estructurales para su reducción han fracasado, negando la validez de la famosa "teoría del derrame" y polarizando la situación social. De allí que la agenda del $\mathrm{BM}$ en los umbrales del siglo $X X \mathrm{X}$ incluy $\theta$ como cuestión clave, según Prats $(1998,13)$ la consideración del rol del Estado y en consecuencia, la Reforma del Estado en función de su eficacia. 
Dedicamos la próxima sección a establecer una primera aproximación a las características más resaltantes de la modernización de la Gestión Pública.

\section{Rasgos básicos de la modernización administrativa del sector público}

Modernizar constituye una aspiración de todas las épocas, para estar a tono con las circunstancias y exigencias del momento contemporáneo y adecuarse a algún modelo de referencia más avanzado-especialmente cuando se trata de los paises subdesarrollados- por lo cual cuando se habla de modernización es necesario precisar su contenido, especialmente si se considera que existe un sin fin de interpretaciones acerca del concepto de modernización, siendo tal vez una de las constantes el uso de modelos del mundo occidental o Primer Mundo.

Para efectos de este trabajo nos limitaremos a partir de las tesis de Omar Guerrero (1992), quien al discutir los alcances de la teoría de la modernización destaca las siguientes cuestiones: a) la modernización constituye un conjunto de relaciones sociales distintas a las precedentes - a las cuales han destruido- que ocurren en un lapso de tiempo y que tienen como escenario fundamental a la sociedad civil (pero que a nuestro juicio, puede ser impulsadas desde el Estado); b) los principales signos de modernización son la sujeción de la sociedad a una organización política especifica, el engranaje de toda la humanidad (al menos de amplias zonas) bajo los criterios de un modelo económico dominante y las organizaciones de la sociedad civil como escenario histórico fundamental; c) no se puede identificar modernización con algún país o continente; d) crecimiento económico no implica necesariamente modernización; e) el desarrollo político, en tanto capacidad de las instituciones políticas para asimilar cambios y protestas, no garantiza una modernización exitosa, si no puede producir condiciones suficientes de subsistencia económica para un pueblo.

En consecuencia, la modernización está inevitablemente ligada al desarrollo económico, político y social. Sin embargo, el concepto de desarrollo también ha tenido diferentes interpretaciones, de la misma forma que han sido diversas las estrategias asumidas o impuestas a los pueblos para lograr el desarrollo. Además, por la importancia que el Estado ha adquirido en los últimos siglos, su modernización se vincula a determinados modelos de gestión estatal.

Durante la década de los sesenta, el desarrollo significaba alcanzar un nivel de vida similar al de los países industrializados; ello implicaba poner énfasis en el crecimiento económico cuantitativo, el cual provocaria avances sociales cualitativos (teoría del derrame); y requería adoptar el modelo burocrático de administración pública occidental (caracterizado por centralización, unidad de mando, prescripción plena, división de funciones y tareas, racionalidad legal), que presuponia formalmente neutralidad politica y probidad, a pesar de la subordinación de hecho a las clientelas externas y a las elites económicas. Para la puesta en marcha del modelo de administración para el desarrollo los países subdesarrollados contaban con el "apoyo" técnico y económico de las agencias multilatera- 
les. Este modelo, fue considerado por Dwivedi y Nef (1982) como de contrainsurgencia, puesto que estaba directamente vinculado a la lucha contra la subversión desbordada en este período por el auge de las luchas de liberación nacional estimuladas por el triunfo de la revolución cubana; debe destacarse que el apoyo técnico y financiero suponía además, ayuda militar para la seguridad interna.

Durante el mismo período se impulsaron otros modelos de administración para el desarrollo, a saber los insurgentes (China, Guinea Bisseau, Cuba), producto de la lucha armada y algunos esfuerzos revolucionarios desde el gobierno (India, Tanzania, Libia). Estos casos, como es lógico suponer, presentan características discrepantes con el modelo contrainsurgente, proporcionales al grado de autonomía con que cada país emprendió su proyecto de desarrollo. Al respecto vale destacar en primer lugar, el esfuerzo consciente por innovar para crear una vía alterna, por experimentar y aprender de los errores, así como el reconocimiento de la vinculación entre política y administración; además, en todos estos países prevalecieron enfoques descentralizados de administración pública con énfasis en la gestión local orientada a la satisfacción de las necesidades básicas, así como mecanismos de participación de la sociedad civil en la gestión estatal (Dwivedi y Nef, 1982).

¿Cuáles son las características de la modernización de la administración pública cuando finaliza el siglo XX y los organismos multilaterales reconocen la necesidad de un reordenamiento orientado a las necesidades básicas conjuntamente con el crecimiento económico, tal como plantea la CEPAL? Del análisis de diversos documentos se deduce que los procesos de reforma de la gestión pública en paises de Europa y América, cuyos objetivos declarados son búsqueda de eficiencia, equidad y profundización de la democracia, pero con énfasis real en la primera, manifiestan las siguientes tendencias:

a. Cambios en la división social del trabajo, con asignación de responsabilidades de gestión pública a la sociedad civil: sector privado y Tercer Sector.

b. Cambio en los sujetos responsables y en el proceso de toma de decisiones y en las relaciones con la sociedad civil: descentralización, negociación y participación.

c. Flexibilización de la organización interna

d. Relaciones laborales con criterios flexibles y meritocráticos

e. Paso de una organización autorreferenciada a otra centrada en el cliente, lo cual resalta el énfasis en la eficacia y la importancia de las estrategias de marketing en el sector público.

f. Cambio del financiamiento a la oferta por el financiamiento según la demanda

g. Uso creativo de los avances tecnológicos en informática: no automatizar lo "viejo"

h. Desarrollo institucional para la consolidación de la cultura necesaria al cambio.

\subsection{Cambios en la división social del trabajo}

La primera tendencia modernizadora consiste en la modificación de los 
criterios de asignación de responsabilidades de gestión pública, estableciendo una nueva división social del trabajo: el Estado conserva la decisión inicial, pero delega en el sector privado y en el Tercer Sector la ejecución, asi como el margen de decisión implícito en todo proceso de ejecución de políticas. Esto responde a la propuesta de la Reingeniería de "traspasar las fronteras organizacionales externas" si fuera necesario, bajo el criterio de que el trabajo se debe realizar en el lugar adecuado; se constituyen asi redes organizacionales, recurriendo a la asociación con otras organizaciones públicas y privadas. Se tiende así a sustituir la organización piramidal por redes de instituciones interrelacionadas que optimizan las economías de escala, la coordinación y la explotación de sinergias. Se declara como objetivo lograr una gestión pública basada en principios de pluralismo y diversidad, que responda adecuadamente a las presiones de la globalización y aproveche el desarrollo tecnológico en informática. Las alianzas y asociaciones se materializan por ejemplo en la separación de funciones de aseguramiento o recaudación y prestación de servicios, o en la realización de acuerdos de abastecimiento con proveedores fijos seleccionados según ciertos criterios; así mismo en el uso de contratistas privadas u organizaciones del tercer sector, para: a) prestación de servicios al ente estatal que no se consideran esenciales a su actividad fundamental; b) obtención de mano de obra sin incorporarla a la nómina de la organización; c) prestación por cuenta del Estado, de aquellos servicios que continúan siendo responsabilidad del gobierno; y d) administración de entes guberna- mentales. El uso de contratistas se considera privatización periférica y constituye también un mecanismo para flexibilizar las relaciones laborales, porque la externalización generalmente degrada las condiciones de trabajo, especialmente en cuestión de estabilidad en el empleo, salarios y jornada de trabajo. Se profundizan de esta manera cambios en la división social del trabajo que ya se venían produciendo en forma discreta. (Therborn, 1979; Osborne y Gaebler, 1994; ; Gomà y Brugué, 1994; Kliksberg, 1994b; Coriat, 1995,98; Hammer y Champy, 1996; Watanabe, 1997; Castells, 1998; Peixoto, 1998,9)

\subsection{Cambios en las relaciones con la sociedad civil}

La segunda tendencia relevante de la modernización de la gestión pública es el cambio en las modalidades y en los sujetos responsables de la toma de decisiones, conjugando la descentralización político-territorial con la administrativa; la descentralización administrativa, entendida como transferencia de competencias a los órganos regionales y municipales, según Mattos $(1990,167)$ se pregona como la nueva panacea para enfrentar los problemas que afectan a las colectividades locales, asignándole objetivos ilusorios de promoción del desarrollo, democratización, incremento de la participación popular y reducción de la injusticia social; si bien la descentralización puede modificar la distribución territorial del poder, no puede generar la transformación de la estructura económica, política e ideológica, especialmente cuando la globalización ha incrementado la movilización de los capitales hacia aquellos luga- 
res donde tengan mayores posibilidades de acumulación; esto significa que para atraer inversión productiva, el poder de decisión local tendría que utilizarse para crear condiciones favorables al capital, las cuales difícilmente serán propicias al desarrollo con sentido humano (Castells, 1998; Mattos, 1990).

Contrariamente a sus virtudes, defendidas tanto por intelectuales neoliberales como progresistas, podría afirmarse que la descentralización constituye una estrategia intermedia que debe facilitar transformaciones más profundas como la privatización bien sea en forma plena, a través de la venta de la propiedad estatal o privatización periférica, la cual utiliza como mecanismos la contratación con el sector privado o con organizaciones del tercer Sector, de la administración de entidades o funciones públicas o de la prestación de servicios. La descentralización crea las condiciones necesarias para la privatización, al quebrar la unidad sindical, limita la acción organizada contra ella, a la vez que regionaliza los conflictos sociales.

Por otra parte, en los procesos de modernización de la administración pública tiende a prevalecer en la toma de decisiones el modelo de interacción frente al de intelección (Oszlak,1984), por lo cual se enfatiza la necesidad de negociación y concertación, así como el reconocimiento formal de la importancia de la participación ciudadana en la gestión y no sólo en la demanda de servicios o ayudas; todas ellas expresan nuevas relaciones del Estado con la sociedad civil tendientes a superar el clientelismo y el formalismo; se considera además que la participación contribuye al logro de la eficiencia y la eficacia de programas sociales, aun cuando no se ha construido suficiente cultura pública ciudadana ni en ef funcionariado para la incorporación efectiva de la participación en la gestión pública. (Fadda, 1990; Dilla, 1994; Kliksberg, 1994b; Osborne y Gaebler, 1994; Cunill, 1997; Blanco, 1997).

El admitir estas nuevas formas de interrelación con los organismos de la sociedad civil, tiene un indudable objetivo legitimador, niega la dicotomía políticaadministración y reconoce de hecho los aportes de los modelos alternativos de desarrollo emprendidos por los gobiernos insurgentes antes mencionados, así como las experiencias locales exitosas basadas en la participación, como el caso de Villa El Salvador en Perú. Sin embargo, la participación ciudadana solo puede ser realizada como una práctica diferencial, puesto que el concepto de "ciudadano" oculta las diferencias sociales entre los distintos estratos sociales (Holloway, 1982; Fadda, 1990; Zapata, 1992; Dilla, 1994).

El potencial de la participación para incidir en la gestión pública depende de algunos factores como los siguientes: a) el contexto en que se produce; b) el grado de organización de los grupos que intervienen; c) el origen de la iniciativa, bien sea surgida de la misma comunidad o promovida por organismos gubernamentales, en cuyo caso existe el riesgo de manipulación; el tipo de participación: consultiva, decisoria, en la ejecución o en el control de la ejecución. 


\subsection{Flexibilización de la organización interna}

La tercera tendencia modernizadora, preconizada fundamentaimente por el sector privado, es la búsqueda de flexibilidad organizacional interna, propuesta para el sector público en términos de simplificación del trabajo. Se trata de modificar los criterios rígidos de división vertical $y$ horizontal del trabajo, por la via de rediseñar los procesos y usar equipos de trabajo polivalentes, dando lugar a estructuras más horizontales y flexibles, que permitan la readaptación de las organizaciones sobre la marcha. Esto exige, de acuerdo a la reingeniería, cambiar la forma de trabajar, alterando significativamente los procesos y organizándose en torno a proyectos o al proceso clave, entendido como conjunto de actividades que recibe uno o más insumos y crea un producto de valor para el cliente. (Kliksberg, 1994b; Hammer y Champy, 1996).

Se deben rediseñar procesos básicos del negocio y no departamentos, siguiendo su secuencia natural, mirando a través y más allá de departamentos funcionales, cruzando las fronteras organizacionales y rompiendo con el trabajo en serie. El trabajo se realiza en el sitio adecuado, centrando la atención en el objetivo de poner los bienes y servicios en manos del cliente que los pidió. Se deben efectuar cambios básicos en el trabajo real que hacen los empleados, combinando varios oficios en una sola persona o en un equipo de caso, que asume la responsabilidad ante el cliente; para procesos más complejos se debe asignar la responsabilidad global a un gerente de caso, que cree la ficción de una responsabilidad única. Al comprimir los proce- sos horizontal y verticalmente, los mismos trabajadores se auto dirigen, controlan y toman decisiones dentro de los límites fijados por las metas y normas de calidad. Los procesos rediseñados pueden tomar diferentes formas; se diseñan múltiples versiones para elegir la adecuada a cada situación; de modo que no hay estandarización. Oficios simples se vuelven complejos, multidimensionales y más substantivos, mientras procesos complejos se vuelven simples (Osborne y Gaebler, 1994; Hammer y Champy, 1996).

El rediseño de procesos, iniciado por los japoneses, es analizado por Coriat (1995) como un modelo que sigue principios opuestos a la clásica organización del trabajo basada en la extrema división horizontal de tareas (que dio lugar a tareas simples y repetitivas) y en la máxima prescripción (división vertical del trabajo). Propugnada por Adam Smith, y refinada por Taylor, Ford, y Fayol, se impuso por más de dos siglos como el modelo ideal de explotación del trabajo incluso en los países socialistas, a pesar de los cuestionamientos de Marx en El Capital, de Gorz, Braverman y otros autores maxistas. El nuevo modelo se empieza a desarrollar a mediados del presente siglo en la fábrica Toyota, por el ingeniero japonés Ohno, como respuesta a diferentes condiciones del mercado: limitaciones financieras, demanda diversificada de cantidades variables del producto y .cada vez mayores desarrollos tecnológicos en informática.

La clave de este modelo, caracterizado por la compresión o simplificación de los procesos de trabajo, integrando en un solo hombre o mujer diversas tareas y conformando equipos polivalentes, es la 
intensificación del trabajo, de acuerdo a las condiciones del actual nivel de desarrollo de las fuerzas productivas. La transferencia del modelo al sector público, bajo el nombre de "simplificación del trabajo" resulta más compleja que en el sector privado; en la industria las máquinas modernas, basadas en el control numérico computarizado, permiten fácilmente la asignación de un obrero para atender varios equipos en forma simultánea.

La flexibilidad en la organización de tareas y funciones exige cambios en los sistemas de control, con disminución de la importancia del control jerárquico tradicional, basado en sistemas clientelares y de vigilancia del proceso, que ahogan la innovación; los nuevos sistemas de control se centran en los resultados, la transparencia y la rendición de cuentas, así como abren posibilidades para el control social. Además, el modelo faculta a los trabajadores para tomar ciertas decisiones menores; los incentivos salariales al equipo polivalente, en contraposición al incentivo económico individual, impulsan a los integrantes del grupo a controlarse en forma recíproca, para evitar fallas $y$ asegurar el bono de productividad correspondiente; esto genera una gran presión y rompe la solidaridad de clase. El énfasis en el trabajo real y no en la burocracia que controla, cuyo trabajo se considera que no agrega valor; transforma la función supervisora la cual debe asumir ahora responsabilidades de capacitación y asesoría antes que de vigilancia, lo cual implica una fuerte eliminación de cargos de gerentes medios y funcionales. Por otra parte, en ciertos trabajos se acepta el control posterior, con tolerancia de abusos limitados. (Osborne y Gaebler, 1994; Coriat,
1995; Hammer y Champy, 1996; Bjur, 1996; Watanabe, 1997).

\subsection{Relaciones laborales flexibles y meritocráticas}

En correspondencia con la flexibilidad organizacional, se asume como prioritario flexibilizar la administración de personal y orientarla por criterios meritocráticos, ámbito en el cual se han concentrado grandes esfuerzos por su efecto racionalizador de costos y depurador de la burocracia. Se trata de pasar de la administración rígida, clientelar y basada en la norma, a otra organización que, en primer lugar, proyecte una imagen de calidad, responsabilidad y eficiencia, estimulada por incentivos según el mérito; y en segundo lugar, sea una organización dúctil, desregularizada, donde prevalezcan la movilidad intra e interorganizacional del personal, la remuneración de acuerdo a la productividad, y la meritocracia en función de la productividad, la innovación y la movilidad. El requisito primario de la flexibilidad laboral es la modificación de los sistemas de contratación por tiempo indeterminado y el ascenso por antigüedad, propios de la burocracia pública, por sistemas de contratación precaria, por períodos fijos, a tiempo parcial y contratación de personal utilizando suministradoras de mano de obra o microempresas formadas por los anteriores asalariados; por lo tanto, la flexibilización laboral siempre va acompañada de procesos de reducción de personal y desregularización.

La movilidad juega un rol clave en la formación de directivos, en primer lugar facilitando el desarrollo de experiencia en diferentes áreas de la misma organización, así como en otros entes del sector 
público; y en segundo lugar, por el intercambio recíproco con el sector privado, lo cual permite el desarrollo de una mentalidad orientada al mercado en los funcionarios públicos. Esto requiere además la capacitación hacia una nueva cultura organizacional, cuestión que desarrollaremos más adeiante. En aras de la nueva cultura surgen criterios adicionales a la capacidad para la selección de personal, tales como el carácter, la iniciativa y la autodisciplina; asi mismo toman fuerza los procesos de capacitación, especialmente en cuanto a los requerimientos necesarios para pasar de la especialización a la polivalencia y al uso de sistemas automatizados; es fundamental la formación del personal directivo, con un peso importante de interacción con el sector privado, bien sea en procesos conjuntos de formación o por la vía de la prestación de servicios profesionales de los funcionarios públicos en el sector privado o en organismos internacionales, asi como la participación de asesores de la empresa privada en altos niveles de formulación de políticas estatales. (OCDE, 1995; Bresser Pereira, 1997; Pratts, 1996 o 1998?).

La introducción del sistema meritocrático en las relaciones laborales implica a) cambios en los sistemas de selección clientelar del personal de la administración pública, preferiblemente recurriendo a la selección externa, contratada con universidades o empresas privadas para evitar el tráfico de influencias; b) creación de incentivos a la productividad, basados fundamentalmente en sistemas de remuneración vinculados al rendimiento individual y/o grupal, asi como relacionados con la contribución o aporte del funcionario. c) desarrollo de sistemas de ascenso de acuerdo a la evaluación del desempeño.

Para la nueva política de personal es fundamental el cambio en las relaciones con las organizaciones sindicales involucrándolas en el proceso de reforma, pero también fragmentando la negociación colectiva; la descentralización, la subcontratación y la colaboración sindical facilitan la aplicación de medidas drásticas contra los trabajadores, como los despidos y reducen su capacidad de lucha al resquebrajar la organización sindical. Según Medeliín Torres $(1994,69)$ "es el signo de la descentralización y la participación que pretende vaciar y desarticular el contenido conflictivo del cambio".

\subsection{Eficacia y marketing}

Otro aspecto de la modernización es el paso de una organización autorreferenciada -centrada en el funcionario y en el cumplimiento de la norma- a una organización centrada en el "cliente" o usuario, el cual debe tener posibilidad de elección para estimular la competencia y por lo tanto la eficacia de los entes que prestan el servicio, bien sean éstos del mismo sector público, del sector privado o pertenecientes al tercer sector. (Osborne y Gaebler, 1994; Cunnill, 1997). Lo anterior conduce al uso de estrategias de marketing, para difundir la información necesaria al nuevo rol de los usuarios como clientes con derechos y obligaciones, pero también para legitimar al estado, vendiendo -con sentido estratégico- una imagen de eficacia y eficiencia de la organización pública. Pero también tiene como objetivo promover los cambios de valores que favorezcan las conductas necesarias al nuevo modelo de gestión, tan- 
to en los funcionarios como en los ciudadanos. En este sentido, el marketing se vincula al cambio institucional, al cual nos referiremos más adelante (Laufer y Burlaud, 1989).

\subsection{Financiamiento según la demanda}

Otra característica fundamental de la modernización de la gestión pública, vinculado al nuevo rol de los ciudadanos, ya no como beneficiarios del Estado de Bienestar, sino como clientes, es la introducción de cambios en las modalidades de financiamiento, en dos sentidos: en primer lugar se asume que los servicios sociales deben ser financiados no sólo, ni fundamentaimente por el Estado, sino también por la sociedad civil. En segundo lugar, se está pasando del financiamiento a la oferta de servicios, vía asignación presupuestaria, al financiamiento de acuerdo a la demanda, que estimula la competitividad y la eficiencia; además, vincula la autonomía con la exigencia de responsabilidades. Con este fin, el estado crea condiciones para que los usuarios paguen los servicios recibidos u otros mecanismos para la transferencia de recursos; de modo que las instituciones públicas, privadas o del Tercer Sector que participan en la prestación directa del servicio público, obtendrán su financiamiento de acuerdo a los servicios efectivamente prestados, según facturación a los usuarios o a entes de intermediación o aseguramiento. (Jaramillo, 1994; Osborne y Gaebler, 1994).

\subsection{Uso creativo de nuevas tecnologías}

Los cambios anteriormente descritos tienen dos requisitos fundamentales: la modernización de los sistemas de información, siempre que no se reduzca a la automatización de viejos procesos y el desarrollo de las instituciones.

El desarrollo tecnológico en informática actúa como "capacitador esencial" permitiendo el desarrollo de redes organizacionales y la simplificación de los procesos, porque posibilita su integración gracias a las facilidades de comunicación a distancia y en tiempo real e interactiva, a las bases de datos en red, y la existencia de programas sofisticados de diseño como el Auto CAD y softwares expertos que permiten a un generalista actuar como especialista en áreas diversas. (Hammer y Champy, 1996). Tal como afirma Castells (1998): "en la última década se ha constituido un sistema tecnológico (telecomunicaciones, sistemas de información interactivos ..., etc.) ... que se constituye como nuevo paradigma operativo en la década de los setenta, y es la base de la globalización de la economía ... un sistema económico articulado giobalmente, funcionando con reglas cada vez más homogéneas entre las empresas y territorios que lo constituyen".

\subsection{Cambios culturales: nuevas reglas del juego}

Como condición esencial para el cambio se impone el desarrollo institucional, el establecimiento y consolidación de nuevas "reglas del juego" que se incorporen a la cultura organizacional y social, indispensables para el funcionamiento del "sistema social de mercado". Prats $(1998,17)$ destaca que "las instituciones son normas y reglas (formales e informales) que estructuran la acción social. Son el sistema de constricciones y de incenti- 
vos del comportamiento de los individuos y de las organizaciones .. son las reglas del juego social: ellas determinan no sólo los procedimientos del juego, sino quiénes juegan y quiénes no, con qué equidad o ventaja se practica el juego, etc." lo cual evidencia su importancia cuando se pretende impulsar un cambio como el que constituye la reforma de la administración pública. Es por ello que, según Laufer y Burlaud $(1989,243)$ el marketing público está vinculado a la modificación de conductas, ya que: "Cada vez con más frecuencia, la Administración intenta incidir sobre los comportamientos sociales cuyos efectos colectivos son importantes". Pero además, Prats precisa que el análisis de una institución requiere ante todo averiguar la estructura de actores y de intereses subyacente, a fin de no formular "reformas de papel".

Según Prats $(1998,15)$ "el cambio de un modelo de desarrollo -y dei modelo de Estado correspondiente- no es un mero mejoramiento de la racionalidad instrumental con que funciona. Es un cambio de actores, de poder, de conocimiento, de habilidades y competencias, de modelos mentales, valorativos y de significación", por lo cual la cuestión clave es el cambio institucional, cuya importancia ha sido reconocida por el Banco Mundial.

Desde el punto de vista del funcionamiento interno, lo anterior requiere según Vignolo (1998, 52-70), cambios paradigmáticos que permitan trascender la racionalidad de la acción y lidiar con la complejidad propia del "recurso humano", entenderlo en su dimensión integral como persona cuyas emociones, intuición y factores culturales afectan las decisiones, el liderazgo, la comunicación y el tra- bajo en equipo. Es decir "mirar la acción humana como especificada por la emoción, más que por la razón". Aun cuando Prats (1998) considera que se requiere un "doloroso proceso de aprendizaje", Vignolo aporta evidencia empírica según la cual "el cambio cultural es mucho más fácil de lograr, en tiempos cortos ( 5 a 10 años), que lo que la mayoría de expertos creen, ... siempre que se utilice una estrategia participativa adecuada, genuinamente buscada e inteligentemente encaminada".

Se supone que los diferentes cambios antes descritos deben dar lugar a una organización eficiente, que logre saltos gigantescos en rendimiento, ya que se elimina todo trabajo que no agrega valor y se pone el énfasis en la eficiencia global antes que en la de una dependencia.. De igual forma se trata de orientar las instituciones del Estado hacia una actuación en función del mercado. Estos son los aspectos clave del modelo, cuyo centro implícito de atención está en facilitar de una manera más drástica que en épocas anteriores la acumulación de capital de grandes empresas; por una parte, persigue la reducción de costos de los servicios adecuándose a las restricciones fiscales y por la otra, debe colocar en manos del cliente o usuario el bien o servicio que garantice la realización del mismo, su conversión en efectivo, asegurando ingresos fiscales por medio de tasas antes que a través de impuestos que los empresarios están cada vez más renuentes a pagar. (Hammer y Champy, 1996; Castells, 1998; Osborne y Gaebler, 1994).

Por otra parte, y en forma complementaria a la eficiencia, se trata de obtener organizaciones ágiles, expeditas, dili- 
gentes, flexibles, que faciliten la coordinación entre diversos departamentos e instituciones asociadas en una red, para ajustarse rápidamente a cambios en las condiciones del mercado. En función de lo anterior surge como requisito indispensable la capacidad innovadora, en cuanto desarrollo de una perspectiva amplia que permita mirar en muchas direcciones a la vez, para detectar causas de problemas fuera del radio de las expectativas normales y tomar las medidas del caso. (Kliksberg, 1994b,131; Hammer y Champy, 1996; Castells, 1998).

A continuación intentaremos precisar cuáles de los cambios anteriores revisten mayor prioridad para los organismos multilaterales, especialmente para el BM y el BID y cómo desarrollan su influencia para lograrlos, específicamente en el caso del sistema de salud en Venezuela.

\section{Incidencia de los Organismos Multilaterales en la Reforma en Salud}

Tal como plantean Ochoa Henriquez y otros (1997), desde mediados de los ochenta existian fuertes presiones para que se diera una salida neoliberal a la fuerte crisis en que se encontraba el país. Dichas presiones eran fundamentalmente externas provenientes de los organismos multilaterales, relacionadas con el refinanciamiento de la deuda y la posibilidad de obtener nuevos préstamos; pero también internas, particularmente de sectores económicos y de intelectuales de pensamiento tecnocrático.

Aunque la reforma comienza a avanzar en las empresas públicas direc- tamente vinculadas a la economía de mercado, no logra penetrar la administración centralizada. El primer intento de imponer una reforma neoliberal en Venezuela por parte del presidente Carlos Andrés Pérez enfrenta inmediatamente la reacción popular a los ajustes económicos establecidos como política de choque, a escasas tres semanas de iniciado su mandato. En estas condiciones, la reforma económica y administrativa es retomada con mucha cautela, asumiendo sólo recientemente la propuesta de "estrategia selectiva centrada en nudos problemáticos", formulada desde 1988 por la Comisión para la Reforma del Estado (COPRE). De esta manera, "el paradigma de la gerencia pública trasciende el discurso para instalarse en el aparato público venezolano en el área tributaria ... (por su importancia desde el punto de vista de los ingresos fiscales) en la cual ha avanzado significativamente" (Ochoa et al,.1997).

Sin embargo, el proceso en otras áreas se frena aún más con los dos intentos de golpe de estado de 1992 que finalmente conducen a la salida del Presidente Pérez. No obstante el discurso de solidaridad social y de rechazo a las imposiciones neoliberales fondomonetaristas que llevaron a Rafael Caldera por segunda vez a la presidencia de la república, en forma lenta pero segura su gobierno está adelantando la reforma administrativa justamente en el campo social, siendo seguridad social y específicamente salud uno de los primeros sectores de interés; en esto ha jugado un papel importante la presión de los organismos multilaterales y el desgaste de la capacidad de lucha laboral y social en general. 
La reforma del sistema de salud venezolano ha sido promovida por el Banco Mundial y el Banco Interamericano de Desarrollo, los cuales han adelantado los recursos para dos proyectos: el primero a cargo del BM, relativo al control de enfermedades endémicas, que a su vez incluye elementos importantes de reforma administrativa; $y$ el segundo, otorgado por el BID, específicamente destinado a la modernización y fortalecimiento del sector salud. Estos son los únicos financiamientos multilaterales para salud, en un abanico de préstamos para los sectores social, ambiente, educación agricultura, transporte, energía turismo, preinversión y $R e$ forma del Estado; los cuatro préstamos de ésta última área están destinados a la modernización del SENIAT, del sistema judicial y finalmente la modernización y descentralización del sector público, lo cual sugiere desde ya ciertos centros de interés en cuanto a la focalización de la reforma, vinculados al establecimiento de condiciones de seguridad jurídica para el inversionista. Vale destacar además, que la descentralización y la racionalización de costos constituyen casi una constante en los objetivos de los diferentes préstamos (CORDIPLAN/PDVSA, 1997).

\subsection{Influencia del Banco Mundial}

En 1995 se firmó el convenio de cooperación técnico financiera para respaldar el préstamo №BM/3538 por \$54 millones, entre el Ministerio de Sanidad y Asistencia Social por parte del Gobierno de Venezuela y el Banco Mundial, que involucraba en principio a los Estados Aragua, Falcón, Trujillo y Zulia, como coejecutores del proyecto. La modernización de las estructuras y sistemas de administración, se realiza bajo la asesoría directa del Banco Mundial o de consultores satisfactorios para el mismo, que pueden ser seleccionados por las entidades venezolanas, siempre que se adecuen a las "Pautas para el Uso de Consultores por Parte de los Prestatarios del Banco Mundial como Agencia Ejecutora" publicadas por el BM en agosto de 1981.

De los términos del convenio con el Banco Mundial, destinado básicamente al control de enfermedades endémicas, se desprende el interés por los siguientes objetivos relacionados con la modernización del sistema de salud, cuyo incumplimiento en algunos casos, da derecho al Banco para suspender los desembolsos:

a. Mejorar la relación costo beneficio de los sistemas de salud, a través de la modernización de las estructuras y sistemas de administración.

b. Aumentar la productividad de los recursos humanos, mediante la capacitación, los sistemas de contratación de personal y la remuneración vinculada al rendimiento.

c. Mejorar la cultura en salud de los ciudadanos, mediante campañas de divulgación sistemática que enfaticen la prevención y el uso racional de los servicios de salud.

d. Priorizar los servicios de salud a financiar.

e. Desarrollar programas específicos de seguimiento y evaluación del proceso de reforma.

La modernización de las estructuras y sistemas de administración supone, según el convenio con el $\mathrm{BM}$, redefinir las políticas de salud en cuanto: a) modelo 
alternativo de organización de los recursos humanos e instalaciones; b) modificar el sistema de financiamiento de los servicios de salud; c) descentralizar, fortaleciendo las capacidades de los Estados (regiones) Coejecutores para planificar y gerenciar los servicios de salud; $d$ ) manejo estratégico de la imagen. Todo ello con la finalidad declarada de lograr una prestación de servicios de salud más justa y costo-efectiva, lo cual exige, además, la introducción y desarrollo de sistemas de costo. Si se analiza cada uno de los aspectos de la modernización, es dificil encontrar su relación con la finalidad de una prestación más justa, puesto que el nuevo sistema prevé un conjunto de exclusiones del financiamiento estatal, como veremos más adelante; sin embargo, es posible que sí apunte a una mayor racionalidad, en términos de la relación costo/beneficio.

Por otra parte, el compromiso con el BM supone rehabilitar la infraestructura fisica de hospitales y ambulatorios, así como reemplazar sus equipos básicos. EI BM exige el desarrollo de programas piloto en cada Estado (región) Coejecutor; en el Estado Zulia el mismo se realiza por convenio con el equipo de Tecnología de Procesos Biológicos de la Universidad Simón Bolívar, el cual participó previamente en la rehabilitación del Hospital de Niños J.M.de los Ríos. (Mijares y Lara, 1997).

En cuanto al financiamiento estatal de la salud, el BM pretende que el Ministerio precise "los servicios de salud que deberían calificar prioritariamente para financiamiento por parte del gobierno", incluyendo la identificación de las intervenciones de salud, criterios y metodología para determinar las estrategias para el fi- nanciamiento y la programación para redirigir los gastos públicos hacia aquellos servicios considerados fundamentales, lo cual implica que algunos no serán financiados por el estado y por lo tanto, serán transferidos al sector privado $o$ al Tercer Sector. En consecuencia el BM exige la realización de análisis de costos y el desarrollo de alternativas financieras para cubrir dichos costos en los centros de salud públicos.

Respecto a la descentralización, el MSAS firmó un convenio específico con el BM, de acuerdo al artículo 6 de la Ley Orgánica de Descentralización, Delimitación y Transferencia de Competencias del Poder Público (1989), condicionando rigurosamente la ejecución del préstamo a su cumplimiento. Dicho convenio implicaba la elaboración de un cronograma de transferencia de los servicios de salud al estado coejecutor respectivo, satisfactorio para el banco, incluyendo el personal de salud, recursos presupuestarios y bienes. Igualmente requería un estudio para identificar mecanismos preferenciales para la transferencia de recursos del gobierno nacional a los gobiernos estadales (regionales) y de éstos a las instalaciones de salud, así como el nivel apropiado de recursos a ser transferidos a cada entidad y los compromisos de desempeño entre el ministerio, los Estados y los centros de salud, precisando las metas correspondientes. Sin embargo, más allá de la descentralización de competencias, el objetivo de fondo es otorgar autonomía legal y operacional a hospitales y ambulatorios, para mejorar su administración, la asignación de recursos y la ejecución, cuestión que debía iniciarse con un programa piloto. 
Para el manejo estratégico de la imagen se da mucha importancia al entrenamiento del personal de salud tanto en el MSAS y en los estados (regiones) coejecutores, en manejo de imagen y relaciones públicas, de modo que estén en capacidad de desarrollar estrategias de marketing para "vender" las virtudes de la reforma. Además, precisa el diseño y operación de sistemas de comunicaciones y sistemas para monitorear la opinión pública. Las estrategias de marketing han sido obvias y persistentes respecto a la reforma del sistema tributario, pero aún no se perciben suficientemente en el sector salud, cuya reforma aprobada a fines de 1998 es cuestionada por el nuevo gobierno nacional.

La garantía del cambio deseado se fundamenta en el desarrollo institucional, por lo cual el convenio insiste en la formación de los funcionarios, especialmente los directivos, en el área de negociaciones, solución de conflictos y comunicación efectiva, en correspondencia con los cambios que se están produciendo en cuanto a la nuevas relaciones con la sociedad civil y su participación en la toma de decisiones. Igualmente tiene como soporte el entrenamiento de personal en gerencia básica, con énfasis en control de calidad y productividad; complementariamente incluye el fortalecimiento de habilidades y conocimientos en 10 relativo a participación de la comunidad, legislación laboral, gestión de recursos humanos y mantenimiento.

Otro aspecto al cual se está dando énfasis en los convenios con organismos internacionales es la recuperación y descongestionamiento de los hospitales, fortaleciendo la responsabilidad de los am- bulatorios en la atención de primer nivei y en la prevención de la salud. Para mejorar la gestión de los centros de salud, el BM exige el desarrollo de sistemas de recursos humanos, planificación estratégica, materiales y drogas, mantenimiento de infraestructura y equipos, administración financiera, manejo de desechos sólidos; información epidemiológica e información gerencial para apoyar los anteriores sistemas en los centros de salud públicos; en correspondencia con lo anterior, es indispensable mantener registros adecuados, tanto epidemiológicos como de costos.

Lo expuesto hasta el momento recoge las propuestas del Banco Mundial para la reforma del sector salud, incorporadas en el proyecto sobre control de enfermedades endémicas. A continuación precisaremos la posición del Banco Interamericano de Desarrollo, en el cual descansa el mayor peso de los condicionamientos de la reforma, dado el volumen del apoyo financiero.

\subsection{Influencia del BID}

Según José Agustín Riveros, representante del BID en Venezuela, en octubre 1997 se encontraba en su fase final el procedimiento para otorgar un préstamo de 280 millones de dólares destinados al financiamiento del nuevo esquema de seguridad social, en conformidad con la Ley Marco que entonces se discutía en el Congreso, la cual tue aprobada a finales del mismo año. El $25 \%$ del préstamo corresponde a un convenio de cooperación técnica reembolsable, que financiará leyes, reglamentos, modelos y planes actuariales, económicos, financieros y de mercadeo, estudios situacionales, de preinver- 
sión y factibilidad, así como diseños organizacionales y capacitación del personal; el resto del préstamo $(75 \%)$ está destinado fundamentalmente a la capitalización de las administradoras de cada uno de los cinco subsistemas, a saber: a) pensiones y jubilaciones, b) paro forzoso; c) vivienda; d) salud y e) recreación. El financiamiento incluye el pago de los pasivos laborales (Panorama, 22-10-97).

Se concreta el préstamo BID867/OC-VE por \$150 millones de dólares, para complementar el del Banco Mundial por \$ 54 millones y, como es usual, con el compromiso de un aporte nacional equivalente a los dos anteriores, destinado específicamente al "fortalecimiento y modernización del sector salud". El préstamo tiene como objetivos declarados los siguientes (CORDIPLAN/ PDVSA, 1997):

a. Mejorar la cobertura, eficiencia y calidad de las redes de servicio de salud, a través de la racionalización del gasto en el sector.

b. Desarrollar y fortalecer las instituciones encargadas de la coordinación de la prestación de los servicios de salud pública.

c. Reestructurar el Ministerio de Sanidad y Asistencia Social (MSAS)

d. Mejorar la equidad del sistema de salud, promoviendo la racionalización del gasto público hacia la atención de los grupos de bajos ingresos.

e. Apoyar la descentralización de la prestación de los servicios de salud y el fortalecimiento de las instituciones regionales y locales.

Desde el punto de vista de la Reforma del Estado, el énfasis de las acciones a realizar está en la descentralización y la reestructuración - racionalización, lo cual se supone debe conducir a la ampliación de la cobertura, la calidad y la equidad.

Respecto al objetivo descentralizador, el financiamiento del BID apoya la preparación de los planes estadales (regionales) de salud para el fortalecimiento institucional y la organización de los sistemas regionales de salud. Incluye asistencia técnica y capacitación en las áreas de planificación estratégica y contraloría sanitaria, así como en los siguientes sistemas: vigilancia de salud y control epidemiológico, control de gestión estadal, administración de recursos humanos, administración financiera, información estadística y gerencial, abastecimiento, mantenimiento y manejo de desechos sólidos hospitalarios. Es decir, formación en aspectos clave para el modelo gerencial que se considera necesario y en cuestiones eminentemente técnicas relacionadas con los servicios de salud.

En cuanto al objetivo de reestructuración del Ministerio de Sanidad y Asistencia Social (MSAS), al cual subyace la racionalización, la propuesta del BID consiste en primer lugar en el establecimiento del marco juridico del sector, asi como en las siguientes cuestiones: a) redimensionamiento de las unidades organizativas, reorganización del Hospital Universitario de Caracas; b) ordenamiento de organismos especiales adscritos al Ministerio, manejando alternativas de asociación con otras instituciones públicas y privadas; c) desarrollo de sistemas de control de gestión y de la red de informática; d) desarrollo de programas de prevención, promoción e información en salud; ye) reconversión laboral y diseño de la política 
de formación y administración de recursos humanos.

La importancia de la reconversión laboral en el proyecto se evidencia en el hecho que la mayor parte de los desembolsos son para el pago de pasivos laborales, cuestión que sugiere importantes niveles potenciales de reducción de personal. Además, liama la atención que la Propuesta de Reforma de los Servicios de Salud para el estado Zulia, que desarrolla el Proyecto Salud, destine el Capitulo VI al "Sistema de Microempresas"; el mismo estipula que Fundasalud, como dirección administrativa financiera del sistema, contratará microempresas de servicios para atender las 8 redes en las cuales se distribuye geográfica y administrativamente el Sistema Regional de Salud. Dichas microempresas prestarán cinco (5) tipos de servicios: cuidados primarios de salud, cuidados hospitalarios, administración, mantenimiento y abastecimiento.

La versión (aún incompleta) del Proyecto revisado es lo suficientemente escueta como para no explicitar los mecanismos de funcionamiento ni de contratación o asignación de las microempresas a las unidades de servicios de salud.

Al describir los procedimientos de atención el "Proyecto Salud" declara taxativa y escuetamente que "no habrá indemnización (pago del servicio) por parte del Fondo Regional de Salud si no se cumplen los procesos de evaluación, referencia y contrarreferencia del paciente" $(p, 45)$. Se deduce que el pago a las microempresas será en proporción a los servicios prestados, lo cual implica el mecanismo de financiamiento a la demanda, en contraposición al sistema anterior de financiamiento presupuestario global a los centros de atención para ofertar servicios de salud sin ejercer ningún control de desempeño.

De lo anterior se deduce claramente la intención de privatizar plenamente los servicios de salud. Por 10 tanto, la reconversión laboral implica la flexibilización de las relaciones de trabajo, convirtiendo a los actuales asalariados en microempresarios que obtendrán contratos como personas jurídicas para responsabilizarse por un aspecto determinado del servicio; esto reproduce prácticas que ya tienen más de una década en algunas clínicas privadas, en las cuales por ejemplo, el servicio de emergencia, el laboratorio, el equipo de enfermeras de cirugía, etc., son atendidas por microempresas. De esta forma los respectivos equipos humanos que conforman cada microempresa deben organizarse y controlarse reciprocamente para cumplir las guardias necesarias o asumir el trabajo asignado; los trabajadores convertidos en microempresarios reciben una retribución global que ellos mismos deben distribuir, haciendo las previsiones para seguridad social, vacaciones, etc. En algunos casos sus ingresos dependen del tipo y volumen de los servicios prestados, es decir, remuneración según productividad.

\section{Conclusiones}

El análisis empírico evidencia que todas las tendencias modernizadoras de la gestión pública en el ámbito internacional, exceptuando la flexibilidad de la organización interna, están presentes en forma explicita en las propuestas de reforma del sistema de salud que los orga- 
nismos multilaterales como el Banco Interamericano de Desarrollo y el Banco Mundial, han impuesto como condiciones para otorgar financiamiento al Estado.

Sin embargo, se evidencia el peso de aquellos cambios vinculados más directamente con la adecuación del sistema de salud a una economía de mercado; en tal sentido es fundamental a la estrategia la consolidación de la autonomía operativa y legal de los centros de atención (hospitales y ambulatorios), la reconversión laboral con tendencia a la flexibilización del contrato de trabajo y restricciones al gasto social en salud; esto último sugiere el abandono de la responsabilidad del Estado en la prestación de aquellos servicios que no se consideren prioritarios, y por consiguiente la tendencia a su privatización, así como el cambio de las modalidades de financiamiento de los servicios de salud.

La privatización apenas se menciona de manera casi tangencial, al referirse a la reestructuración del Ministerio, en términos de "manejar alternativas de asociación con otras organizaciones públicas y privadas"; pero subyace a la necesidad de que los centros de salud posean autonomía legal y operativa y se materializa en el Proyecto Salud, desarrollado con la asesoria avalada por los organismos multilaterales, al proponer el sistema de microempresas que serán contratadas para prestar todo tipo de servicios. Por lo tanto, la insistencia en la descentralización, está vinculada directamente al hecho de constituir una estrategia intermedia para la privatización, así como para la reducción de personal y la neutralización de los sindicatos, como potencia- les oponentes a la reforma, especialmente en cuanto afecta a los trabajadores.

Por su parte, la reconversión laboral es el nombre formal que reciben los cambios tendientes a flexibilizar las relaciones laborales, cuya condición previa es la reducción de personal, con la finalidad expresa de modificar los sistemas de contratación; es evidente que la via fundamental es la conversión de los trabajadores en microempresarios; esto elimina totaimente las obligaciones legales $y$ contractuales con el personal, puesto que pierden la condición de asalariados y por lo tanto la protección legal correspondiente.

Se supone que los diversos aspectos de la reforma promovida por los entes multilaterales, debería presionar hacia la eficiencia. Sin embargo, la experiencia de otros paises latinoamericanos sugiere que ellos no la garantizan, puesto que la delegación al sector privado y al Tercer Sector se ha convertido en fuente de corupción; además, no aseguran la equidad ni la universalidad en la prestación de los servicios de salud.

\section{Agradecimientos}

La fundamentación teórica de este trabajo es producto de las discusiones mantenidas en el seminario sobre Modernización de la Gestión Pública, realizado en el Doctorado en Ciencia Política bajo la dirección de la doctora Haydée Ochoa Henríquez, en la Facultad de Ciencias Jurídicas y Políticas de la Universidad del Zulia. Igualmente, la investigadora ha contado con el apoyo financiero del Consejo de Desarrollo Científico y Humanístico de esta Universidad. 


\section{Bibliografía citada}

Bjur, Wesley (1996) de la Reingeniería a la Readministración. En busca de un nuevo paradigma. Rev. Venezolana de Gerencia, Año 1, № 1, Maracaibo.

Blanco, Carlos (1997) El Proceso de descentralización en Venezuela. Análisis crítico. B.I.D. Págs. 19-27.

Braverman, Harry (1978) Trabajo y Capital Monopolista. Editorial Nuestro Tiempo, México.

Bresser Pereira, Luis Carlos (1997) Una Reforma Gerencial de la Administración Pública en Brasil. En Rev. Reforma y Democracia № 9, Págs. 61-102.

Castells, Manuel (1998) Hacia el Estado red? Globalización económica e instituciones políticas en la era de la información. Seminario Internacional Sociedade ea Reforma do Estado. Sao Paulo.

COPRE (1988) La Reforma del Estado, Editorial Arte, Caracas.

CORDIPLAN/PDVSA (1997) Guia de Proyectos Públicos con Financiamiento Multilateral, Caracas.

Coriat, Benjamin (1995) Pensar al revés: trabajo y Organización en la Empresa Japonesa, Sigio XXI, 98-123.

Cunill Grau, Nuria (1997) Repensando lo Público a través de la Sociedad. Nuevas formas de Gestión Pública y representación Social, CLAD - Nueva Sociedad, Caracas, Pags. 71-96.

Dilla, Haroldo; Gerardo González y Ana Teresa Vicentelli (1994) Participación Popular y Desarrollo en los Municipios Cubanos. Editorial Tropykos, Caracas, Pgs. 57-104.

Dwivedi, O.P y Jorge Nef (1982) Continuidad y Crisis en la Teoría y en la Administración para el Desarrollo: Perspectivas de los Países Desarrollados y del Tercer Mundo. En Administración Pública: Perspectivas críticas. ICAP.

Fadda, Giulietta (1990) La Participación como Encuentro: discurso político y praxis urbana. Fondo Editorial Acta Científica Venezolana y Universidad Central de Venezuela, Caracas, Cap. I.

Gobernación del Estado Zulia (1996) Modernización de la Gobernación del Estado Zulia. PNUD-FONVIS, Documento del Proyecto VEN/96/011, Maracaibo.

Gornà Ricard y Quim Brugué (1994) Nuevos Modelos de Gestión y Organización Pública. Rev. Autonomies № 18 , Barcelona.

Gorz, André (compilador 1977) Crítica de la División del Trabajo. Editorial Laia, Barcelona.

Gournay, Bernard (1989) Tendencias Actuales del Control de las Finanzas Públicas. Rev. de Control Fiscal, N²5, Caracas.

Guerrero, Omar (1992) El estado en la Era de la Modernización. Plaza y Valdés Editores, México.

Hammer, Michael \& James Champy (1996) Reingenieria. Editorial Norma, Bogotá, Págs. 53-87.

Holloway, John (1982) Fundamentos Teóricos para una Crítica Marxista de la Administración Pública, Ediciones INAP, México.

Jaramillo, Iván (1997) El Futuro de la Salud en Colombia. La puesta en marcha de la Ley 100, Fescol, F.R.B., Fundación Corona, Tercer Mundo Editores.

Kliksberg, Bernardo (1994a) Pobreza. Un tema impostergable. Nuevas respuestas a nivel mundial. CLAD, PNUD, Fondo de Cultura Económica, México. 
Kliksberg, Bernardo (1994b) El Rediseño del estado para el Desarrollo Socio-económico y el Cambio: una agenda estratégica para la discusión. Rev. Reforma y Democracia № 2, Caracas.

Kliksberg, Bernardo (1997) Hacia una Gerencia Social Eficiente. Algunas cuestiones claves. Rev. Venezolana de Ciencias Sociales, Vol.1 № 1, Cabimas, Venezuela.

Laufer, Romain y Burlaud, Lain (1989) Dirección Pública: Gestión y Legítimidad. Instituto Nacional de Administración Pública, Madrid.

Lichsttensztein, Samuel y Baer, Mónica (1987) Fondo Monetario Internacional y Banco Mundial. Estrategias y Políticas del poder Financiero. editorial Nueva Sociedad, Caracas.

Marx, Carlos (1977) El Capital. Crítica de la economía política. Vol.1 Editorial Fondo de Cultura Económica, México.

Mattos, Carlos de (1990) La Descentralización ¿Una nueva panacea para impulsar el desarrollo local? Rev. Economía y Sociedad № 3 165-178.

Medellín Torres, Pedro (1994) La Modernización del Estado en América Latina: entre la reestructuración y el reformismo. Rev. Reforma y Democracia № 9, Caracas.

Mijares, Rodrigo y Lara Estrella, L.O. (1997) Establishment of a Clinical Engineering Department in a Venezuelan $\mathrm{Na}$ tional Reference Hospital. Journal of Clinical Engineering, July/August 1997.

MSAS/BM (1995) Convenio de Préstamo № BM/3538-OC-VE.

OCDE- Ministerio para las Administraciones Públicas (1995) Flexibilidad en la Gestión de Personal en la Administración Pública, INAP, Madrid, Pags. 9-143.
Ochoa, Haydée; Maira Montilva y José Garcia (1997) Marketing en la Administración Pública Venezolana. El caso de la administración Tributaria.

Osborne, David y Ted Gaebler* (1994) La Reinvención del Gobierno. La influencia del espiritu empresarial en el sector público, Editorial Paidós, Barcelona.

Oszlak, Oscar (1984) Políticas Públicas y Regímenes Políticos: Reflexiones a partir de algunas experiencias latinoamericanas. En Documentos del CLAD, Vol. 1 № 1, Caracas, Págs. 8-54.

Peixoto de Albuquerque (1998) Las Estrategias Empresariales y el Nuevo Ambiente Económico. La realidad virtual del cambio. Rev. Venezolana de Gerencia, Año 3 N25, Maracaibo.

Pérez Salgado, Ignacio (1997) El Papel de la Cooperación Técnica Internacional en el Proceso de Modernización del estado y de la Gestión Pública. Reforma y Democracia $N^{0} 8$, Caracas.

PNUD/FONVIS/GEZ (1996) Proyecto de Modernización del Gobierno del Estado Zulia, VEN/96/011.

Prats Catalá, Joan (1996) El Nuevo Juego y sus Reglas. Construyendo la nueva institucionalidad en la América Latina de los 90. Doc. a solicitud de PNUD Cap. I.

Prats Catalá, Joan (1998) Administración Pñublica y Desarrollo en América latina. Un enfoque neoinstitucionalista. Rev. Reforma y Democracia N111, Caracas.

Saravia, Enrique (1995) Procesos de privatización en Argentina y Brasil. Consecuencias en materia de desempleo empresarial y mercado de trabajo. Rev. Reforma y Democracia N ${ }^{9} 4$, Caracas.

Sistema Regional de Salud Zulia (sf) Proyecto Salud, Maracaibo. 
Taylor, Frederick y Fayol, Henry (1983) Principios de la Administración Científica, Editorial Herrero Hnos. Págs. 1935.

Therborn, Göram (1979) ¿Cómo Domina la Clase Dominante? Siglo XXI Editores, México, Pags. 49-148.

Vignolo Friz, Carlos (1998) Nuevos Paradigmas en Management. Una aproximación desde la experiencia de modernización de los sistemas públicos de salud en Chile. Reforma y Democracia № 11, Caracas.
Watanabe, Ben (1997) ¿Pensar o al Revés? Una visión crítica. Rev. Periferias, Año 2 N22, Buenos Aires.

Zapata, Gastón Antonio (1992) Una Estrategia de Desarrollo Alternativa basada en la Participación Social y la Organización Comunitaria. La Experiencia de Villa El Salvador. En ¿Cómo Enfrentar la Pobreza? Aportes para la Acción, Compilado por Bernardo Kliksberg, CLAD-PNUD, Grupo Editor Latinoamericano, Buenos Aires, Págs. 225234. 